\title{
WOODEN TRUSSES RECONSTRUCTION AND ANALYSIS THROUGH PARAMETRIC 3D MODELING
}

\author{
D. Prati ${ }^{1}$, G. Zuppella ${ }^{2}$, G. Mochi ${ }^{1}$, L. Guardigli ${ }^{1}$, R. Gulli ${ }^{1}$ \\ ${ }^{1}$ DA - Dipartimento di Architettura, Università di Bologna, Bologna, Italy, \\ (davide.prati5, giovanni.mochi, luca.guardigli, riccardo.gulli)@unibo.it \\ ${ }^{2}$ DA - Dipartimento di Architettura, Università di Bologna, Bologna, Italy, giulia.zuppella@studio.unibo.it
}

\section{Commission II}

KEY WORDS: Building heritage, Terrestrial Laser Scanning, Generative algorithms, Structural systems reverse engineering, Cloud to $3 \mathrm{D}$ model comparison,

\begin{abstract}
:
This paper aims to indicate a new methodological approach, based on generative algorithms, to attempt a more in-depth and transversal understanding of the behavior of these wooden structures. The developed method accelerates modeling procedures and brings on new tools for analyzing these structural systems when surveyed through TLS devices. The main topic of this work is the most recent step of a wider research project that has analyzed a few wooden roofing structures in the area of bologna. These case studies are represented by a set of important churches in Bologna, all built between the 16th and 18th centuries, whose pitched roofs are supported by timber trusses. Among them, the most impressive is the wooden truss in the St. Peter Cathedral that has approximately 26 meters of span, 7 meters of height for nearly 9 tons of weight. It also shows a complex static conception with the coexistence of an external nondeformable triangle and an internal virtual discharging arc. The focus is on the transformation of the point cloud into 3D models using parametric modeling tools such as Grasshopper generative algorithms. These algorithms, once created for a single truss, allow to automatically generating $3 \mathrm{D}$ models of all trusses, changing only input parameters.
\end{abstract}

\section{INTRODUCTION}

The analysis of the wooden roofing structures is widely present in scientific literature in many contributions. On the one hand, the theme is dealt with in the field of building science and construction technology, and in that of practical technology and restoration. On the other, only a few studies systematically investigated the real behavior of these construction systems counting on practical experience and on-site diagnosis. This is justified by various factors, mainly that roofing structures are hidden and difficult to access, not attracting the attention of technicians and researchers, unless in case of severe problems of deterioration or damage with consequences on people's safety. The specialist literature usually simplifies the constructive conception and static scheme of these artefacts which, even when they stand still to date, show the signs of a long life and frequent changes of configuration over time. The roof is obviously the area of the building that firstly bears accidental loads due to atmospheric phenomena, such as snow, and is most subject to deterioration and fire. Therefore, roofing structures are often characterized by strong transformations and large substitutions. The theme is therefore downgraded to an issue of minor importance for restoration, since the matter of study is no longer original or difficult to date. In other words, it seems to pertain to the art of building and not to the theory of construction. For this reason, it eludes scientific and analytical interpretations, if not at the cost of large approximations.

The main topic of this work is the most recent step of a wider research project that has analyzed a few wooden roofing structures in the metropolitan area of Bologna. These case studies are represented by a set of important churches, all built between the 16th and 18th centuries, whose pitched roofs are supported by timber trusses. The most interesting structures belong to the Cathedral of Saint Peter, the church of San Salvatore Maggiore, the church of San Giovanni in Monte, the Basilica of San Petronio and the Basilica of San Domenico. These buildings are all built in the same period and some of their naves have unusually big dimensions. In fact, Saint Peter's trusses were possibly the biggest in Italy - and perhaps in Europe - at the time of construction. Having approximately 26 meters of span, 7 meters of height for nearly 9 tons of weight it shows a complex static conception with the coexistence of an external nondeformable triangle and an internal virtual discharging arc. Therefore, due to their importance in terms of dimension and structural conception, the case study chosen to be presented in this paper is the church of Saint Peter, the cathedral of Bologna. The analysis of all the case studies allowed to set up a protocol in order to systematize the behavioral assessment of wooden trusses in terms of displacements and deformations. This research protocol includes an acquisition step followed by the digital rendering of the acquired data. This last step provides useful output (photos, drawings, graphs and models) that constitutes the operative tools to define and correctly interpret the behavior of the analyzed constructive systems. Results interpretation therefore allows us to suggest the most coherent design solutions, disengaging them, where necessary, from usual and standardized techniques (Prati et al., 2016) (Figure 1).

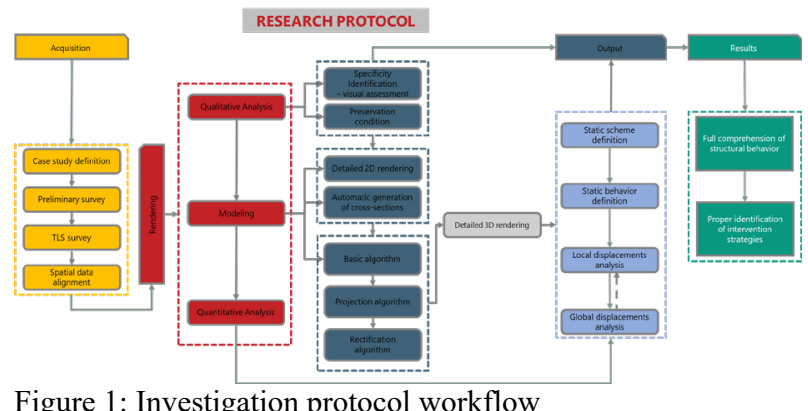

The focus of this paper is mainly on the transformation of the point cloud into 3D models using parametric modeling tools such as Grasshopper ${ }^{\circledR}$ (Robert Mcneel \& Associates, 2018a) generative algorithms. These algorithms, once created for a single truss, allow to automatically generate $3 \mathrm{D}$ models of all trusses, changing only input parameters. Different generated 3D models can be used to simply reconstruct the roofing, using CAD 
or BIM software, or they can be exploited to make comparisons with the point cloud itself in order to highlight deformations and displacements.

\section{RELATED WORKS}

In construction history timber trusses are the main structures of wooden roofs. They are commonly used to cover big halls and, particularly, the naves of the churches. These types of structures are conceived both as planar and tri-dimensional systems and realized with girders of solid wood of different sections, depending on the roof span. The ability to redistribute the vertical load without producing horizontal thrust on lateral walls gave success to these structural configurations since the beginning of the IV century.

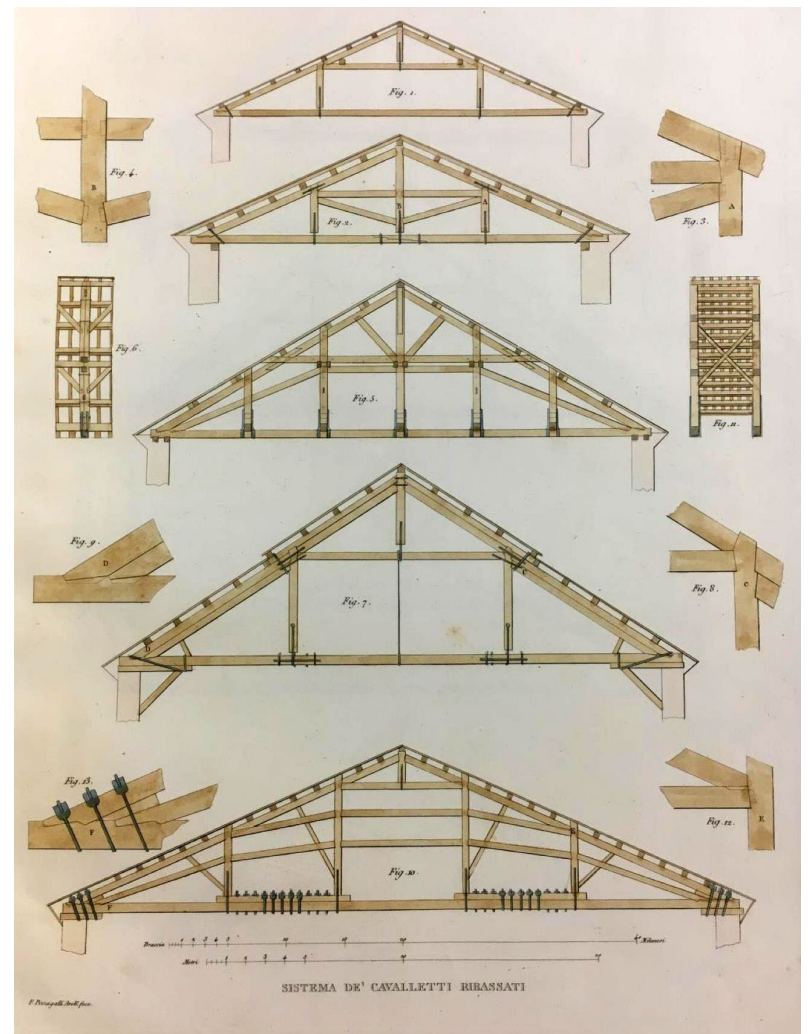

Figure 2: Pizzagalli \& Aluisetti: Tav. XI, Wooden trestle systems

Studies on wide span roofing, especially trusses, are usually based on important textbooks and manuals since the XIV century. Early drawings from Giuliano da Sangallo (Borsi, 1985) were found representing some trusses static scheme. Later on, in the XVI century it is possible to find out sketches from Giorgio Vasari and Sebastiano Serlio (Serlio, 1987) documenting the attention of architects in trying to comprehend these construction systems. Actual classification proposed in literature is the outcome of the XIX century technical sensitivity, that used to force complex and partly structurally undetermined systems into simplified and calculable schemes. The main textbook about wooden constructions is the "Traité de l'art de la charpenterie" written by Armand Rose Émy published in 1841 (Émy, 1841) but in those years, following the positivistic effort of organizing and cataloguing the technical knowledge, a lot of authors published collection of drawings representing many wooden trusses schemes and tried to explain its static behavior (Pizzagalli and Aluisetti, 1827) (Figure 2).
In the recent past, some historians of architecture and construction used to identify structural systems types within these constructive systems, highlighting their behavior based on strongly simplified theoretical hypotheses (Munafò, 2002; Valeriani, 2006). The logic was based most of the times on the idea of forcing the behavior of these structures into well-known or typical schemes.

Nowadays, the awareness that shaping of wooden trusses eludes most of these simplified hypotesis but follows a very precise and often ignored construction practice made clear that retracing the original logic of static schemes and junctions is a de facto nontrivial procedure. Their behavior depends, in fact, on a series of factors: different ways of assembling the linear elements to form longer tie rods or rafters with larger cross section, more or less accentuated stiffness of the knots, due to the carving of notches and to fastenings, presence of more or less brackets and other characteristic details (Guardigli et al., 2015; Zamperini, 2015, 2013).

In the past few years, 3D as-built models for construction quality assessment have been developed using data acquisition techniques like 3D Terrestrial Laser Scanning (TLS) and photogrammetry. However, certain limitations like nonaffordability, requiring expertise to operate, and dependence on time-consuming processes are restricting their usage (Kalyan et al., 2016). Actually, if use of TLS for surveying complex architectural surfaces is not new and is matter of many researches in restoration field (Bello Caballero et al., 2017), rarer, or even absent, is its use applied to the study of hidden spatial structures such as wooden roofing. One example is the analysis of past intervention in the timber roof of San Valentino Castle (BertoliniCestari et al., 2015) in which a TLS point cloud is manually rendered to obtain a finite element model useful to the numerical assessment of the roofing.

Working without resorting to a 3D model may be a way and a lot of scientific literature deals with cloud to cloud comparison and mesh to cloud comparison (James et al., 2017; Lague et al., 2013). However, since one of the goals of the protocol is the assessment of the actual condition of trusses, the comparison between the surveyed point cloud and a theoretical model of the truss itself at the time of construction, can only be made with a 3D model rendered upon strong and resonable assumptions in terms of displacements and deformations of each wood girder over time (Prati et al., 2018).

\section{THE MODEL GENERATION PROCEDURE}

Regardless of current approach to the problem of preservation of historical roofing structures, digital surveying technologies allow extrapolating new and original considerations that would be almost impossible following traditional methods of investigation, based on direct observation or on simplified architectural survey. The outline hypothesis was to exploit the large amount of geometric data, which can be acquired by the TLS device as 3D point clouds, in order to read wooden trusses in detail and then gather specific and comparative information on their static behavior and on their conservation status.

Large and detailed geometric information provides very interesting data to perform an accurate $3 \mathrm{D}$ rendering of each truss into a single roofing but also to evaluate even slight differences in the static schemes of different wooden trusses used to cover different roofs. Furthermore, the accurate modeling of the entire wooden roofing should became the starting point for monitoring future modifications of these structures in terms of displacements during their lifecycle.

Usual modeling techniques, like vectorization of orthophotos or direct 3D modeling using CAD or BIM software, strongly depend 
on the operator and have a low degree of repeatability and precision. Given the size of these artifacts, moreover, it is not possible to resort to the automatic generation of meshes, since the point clouds are largely incomplete and extremely articulated.

To avoid operating with excessive approximations, it was decided to transform the point cloud into 3D models using parametric modeling tools such as Grasshopper ${ }^{\circledR}$ generative algorithms. This procedure allows saving a considerable amount of time and eliminates many of the inaccuracies identified by adopting $3 \mathrm{D}$ point cloud meshing and it is consistently faster than manual modeling methods.

Once identified the case study, in this case St. Peter's Cathedral in Bologna, an accurate TLS survey was carried out. The entire TLS survey of Saint Peter's roofing has been carried out with a FARO CAM2 FOCUS $3 D{ }^{\circledR}$ laser scanner using a targetless approach. The survey campaign took 5 working days and it was necessary to shoot more than 100 scans with a resolution of $12 \mathrm{~mm} / 10 \mathrm{~m}$ and a quality filter of $3 \mathrm{x}$ in order to get the whole roofing. The alignment was performed with the software FARO SCENE 2018® (Faro Technologies, 2018) using an interactive Cloud to Cloud registration. With a medium overlapping between scans of $32 \%$ it was possible to achieve an extremely accurate alignment with standard deviation between corresponding points of $1.2 \mathrm{~mm}$ and a maximum deviation of 1.8 (Scene 7.1 User's Manual, 2017).

The main goal was to exploit the application of parametric modeling, with the creation of a BASIC 3D MODEL, which represents the current condition of the structure. The secondary goal was the creation of the IDEAL 3D MODEL, made through a series of automatized operations, in order to reconstruct a hypothetical original condition of the wooden truss. This second 3D model will be used to be compared with the current state point cloud in order to evaluate displacements and deformations. The generative algorithm has been divided into several sub-phases, in order to guarantee easier management and, above all, a greater understanding of the operating process adopted (Figure 3).

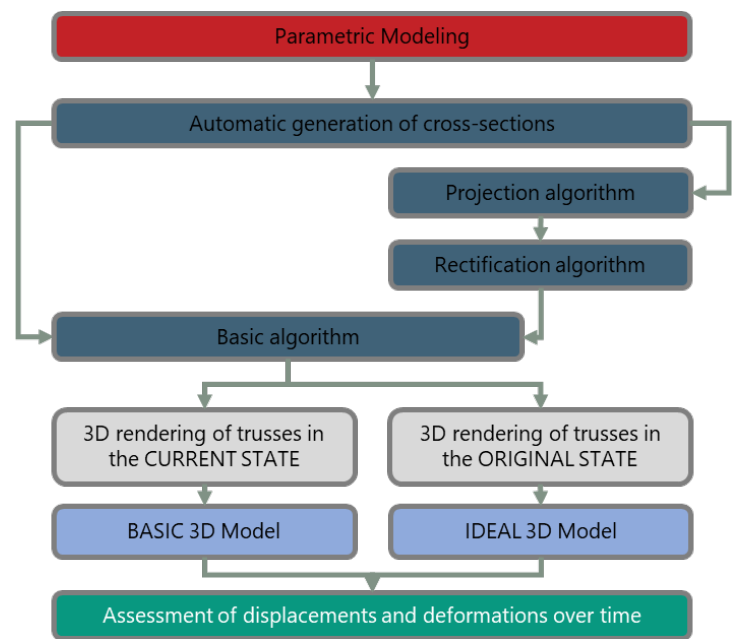

Figure 3: Parametric modeling procedure workflow

\subsection{Automatic generation of cross-sections}

Cross-sections generation was performed starting from the clipped point cloud of a single truss. By clipping $3 \mathrm{~cm}$ wide slices of points near the joints of girders in the truss, it was possible to isolate 2 cross-sections for each element (Figure 4).

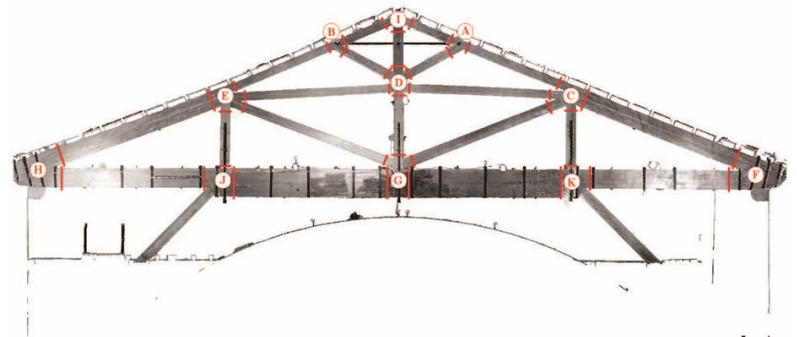

Figure 4: Clipped truss from St. Peter's point cloud. Extracted cross sections are highlighted in red.

Once created these clipping boxes, enclosed points were exported in .xyz format. Exported files were then imported into Geomagic Studio ${ }^{\circledR}$ (3DSystems, 2014a). Using the "Feature - Plan" command for each group of points representing a single crosssection, average plans were created: in other words, the software identifies the plane that best suits the chosen points. Then using the "Curves - Create by Section" command, points of each crosssection were automatically vectorized by using previously created planes as alignment planes.

Vectorized cross-sections were imported into Rhinoceros ${ }^{\circledR}$ (Robert Mcneel \& Associates, 2018b) and an algorithm was created with Grasshopper ${ }^{\circledR}$ plugin to automatically divide these cross-sections into 80 equal-length segments. At last, a new curve was created as interpolation of the 80 segments. The new curve obtained matches almost exactly the input one, since a rather high number of points has been chosen: further increasing this parameter will therefore bring a higher correspondence (Figure $5)$.
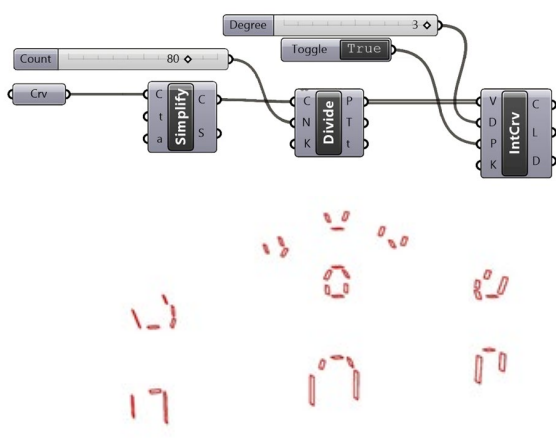

Figure 5: Automatic generation of cross-sections algorithm

\subsection{Basic algorithm}

This algorithm allows to obtain, starting from previously generated curves, the BASIC 3D model of the truss. The "loft" command has been applied to the cross-sections belonging to the same wood girder. This procedure allows the creation of a surface through only the extremity input sections (Figure 6).

Once the "loft" operation was completed on each element of the truss, as the image above clearly shows, it was necessary to complete the modeling of joints. Adopted procedures were differentiated according to the different connection between elements. A specific algorithm was then created for closing the tie-beam in correspondence with the change in cross-section size, for the modeling of the rafters-straining beam joint, for lateral bearings and for the connection between the king post and struts. 


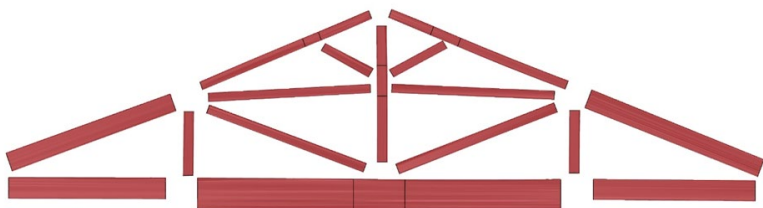

Figure 6: From cross-sections to creation of elements of the truss through the "loft" command

Once the "loft" operation was completed on each element of the truss, as the image above clearly shows, it was necessary to complete the modeling of joints. Adopted procedures were differentiated according to the different connection between elements. A specific algorithm was then created for closing the tie-beam in correspondence with the change in cross-section size, for the modeling of the rafters-straining beam joint, for lateral bearings and for the connection between the king post and struts. For example, for closing the ridge, the end cross-section of the left rafter was translated at the intersection between its axis and the vertical plane passing through the central king post. The curve was then rotated parallel to this plane, becoming the new ridge cross-section. The final portion of rafters was created using the "ruled surface" command. At last, the top cross-section of the king post was extruded up to the center of gravity of the new ridge cross-section (Figure 7).

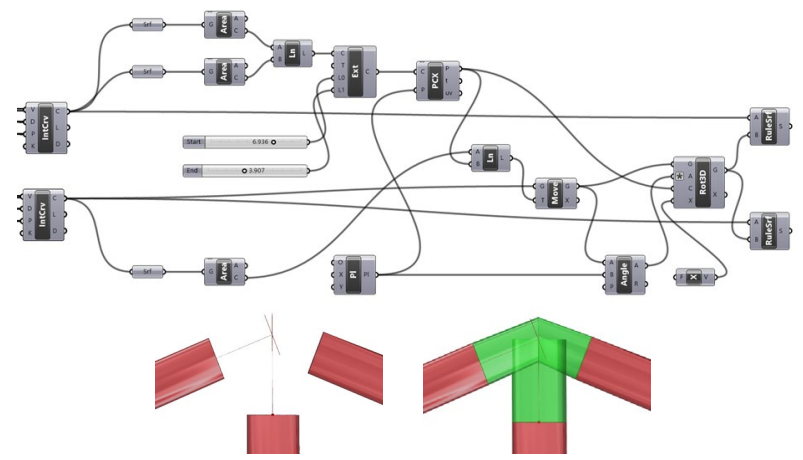

Figure 7: Part of the algorithm for the ridge completion

After completing automatic modeling of all joints, wood girders of the truss were joined together using the "bake" command on Rhinoceros ${ }^{\circledR}$ creating the so-called BASIC 3D Model (Figure 8).

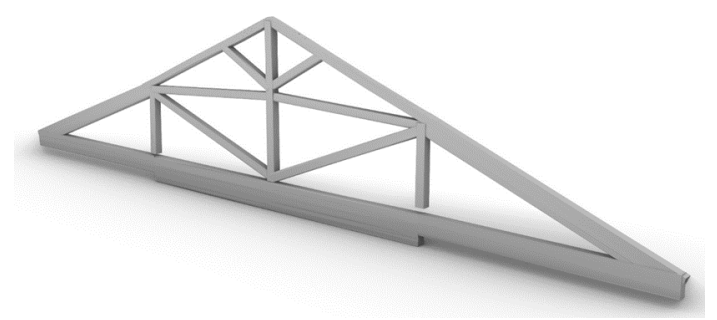

Figure 8: Basic 3D model

To verify the degree of compliance of the rendered model with the surveyed point cloud, a comparison of these two elements is carried out using the software Geomagic Control ${ }^{\circledR}$ (3DSystems, 2014b). This software detects and reports in a chromatic scale distances between the point cloud, considered as reference object, and the BASIC 3D model, considered as test object. Furthermore, it is possible to extract summary reports indicating the percentage of points within the nominal overlap threshold of the two compared objects (Figure 9).

The highlighted row contains the points within the threshold \pm 5 $\mathrm{mm}$ whose deviation is considered non-significant, then treated as correctly overlapping points. The percentage of points within the threshold indicates that the total degree of overlap between point cloud and $3 \mathrm{D}$ model is on average equal to $70 \%$ for all modeled trusses.

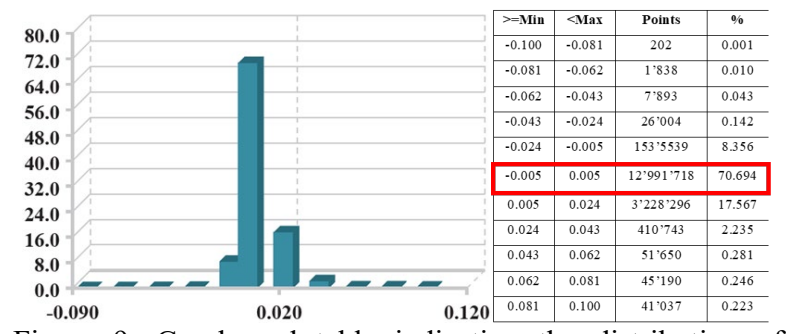

Figure 9: Graph and table indicating the distribution of distances between the point cloud and the basic 3D model

\subsection{Projection algorithm}

The following phases are aimed at the realization of a IDEAL 3D model of the analyzed trusses: the goal was to create a series of algorithms that reconstruct a hypothetical initial configuration, recovering the deformations withstanded by these wooden structures over time.

The first step therefore consists in the generation of the "projection algorithm", which eliminates the rotation of the whole truss outside its plane. This algorithm is applied after the "automatic generation of cross-sections" to the vectorized curves created from clipped points of the point cloud.

Once creates the barycenter of each cross-section, the vertical plane passing through the barycenters of the two external curves of the tie-beam is created. This operation is based on the hypothesis that the lateral bearings of the tie-beam are still in their original position or withstanded negligible movements during the whole life of the roofing, in particular along the longitudinal axis of the building (Figure 10).
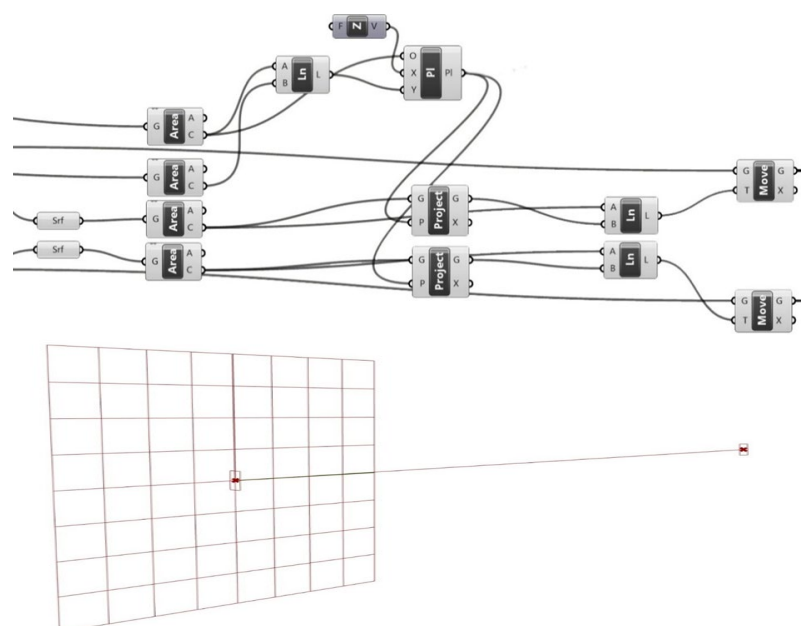

Figure 10: Algorithm for the projection of cross-sections on the vertical plane

The barycenter of all other cross-sections is projected on this vertical plane using the "project" command. The connecting lines between the original barycenters and the new ones are used as laylines for the "move" command. This operation simply moves the original curves from the old barycenter on the projected one. In other words, this algorithm operates the projection of the curves, by moving their center of gravity, in correspondence with the plane passing through the axis of the tiebeam. A similar procedure is applied to the lower cross-section of the queen posts which are free to rotate in the plane of the truss. 
Their projection plane is built using the upper sections barycenter of the queen posts themselves and their lower sections are projected on this plane (Figure 11).

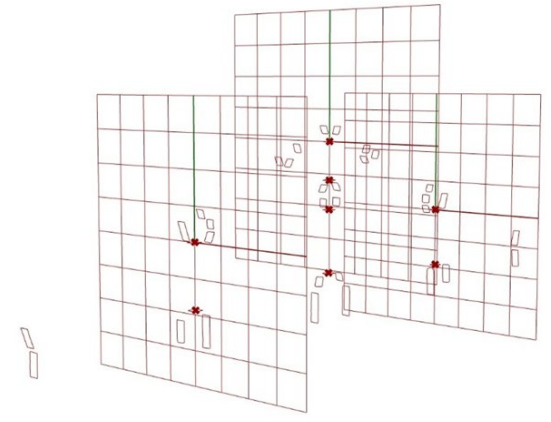

Figure 11: Projected curves on the vertical plane

\subsection{Rectification algorithm}

The last phase identified for the creation of the IDEAL 3D model of trusses is essentially focused on the reconstruction of the main undeformed external triangle, consisting of tie-beam and rafters. This last step therefore consists in the generation of the "rectification algorithm", which eliminates the bending of all wood elements of the truss. In particular, the aim was to highlight deformations and displacements these elements underwent and to try to eliminate them, redefining, at the same time, the positions of the king and queen posts and struts. By reconstructing the ideal lines joining the extreme sections of the various elements, it has been found out that the tie-beams also show signs of flexural deformation. The procedure is therefore developed according to the following steps:

1. Flexural deformation of the tie-beam is eliminated moving up all its cross-sections till the new rectified axis;

2. All cross-sections connected with posts and struts are moved up of the same distance, due to the hypotesis of conservation of the angle between tie-beam and rafters;

3. Flexural deformation of the rafters is eliminated moving up all its cross-sections till the new rctified axis;

4. All cross-sections connected with posts and struts are moved up of the same distance, due to the hypotesis of rigid connection of the joints between rafters, straining beam and queen posts.

Following the order of application of previously defined algorithms, cross-sections have been vectorized and optimized and subsequently projected into the tie-beam plane. Rotations of queen posts inside the truss plane were also eliminated.

Starting from the new position of the curves representing the cross-sections modified up to now, the next step is therefore to create a straight line joining the two curves closest to the lateral bearings of the tie-beam. Thereinafter, cross-sections of all the posts and of all girders connected to them have been raised (Figure 12).

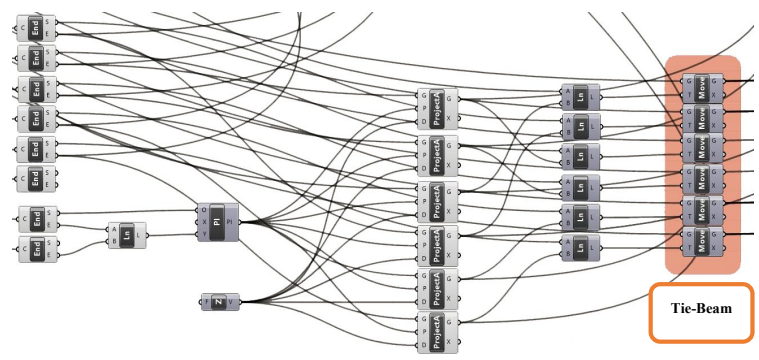

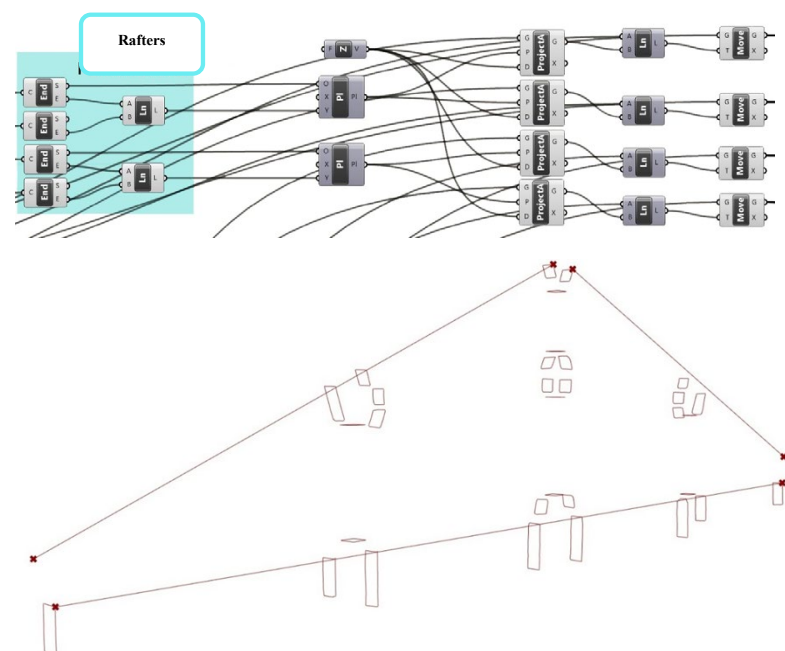

Figure 12: Parts of the algorithm for the rectification of trusses

Once this operation was completed, the reference lines for the rectification of the rafters have been created. This line is generated connecting the cross-sections closest to lateral bearings, left unmodified by previous algorithms, and the crosssections near the ridge, that have been previously raised. All other curves belonging to the rafters have therefore been moved up till the new reference line. Once al the operations described above have been carried out and the curves have been moved in the new position, all cross-sections of all wooden girders of the truss reached their final position, the so-called "rectified" position.

After performing all the procedures for the "automatic generation of cross-sections" and subsequently running the "projection" and "rectification" algorithms, all wood girders of the truss can be modeled using again the "basic algorithm". At the end, as for the creation of the basic 3D model, using the "bake" command on Rhinoceros ${ }^{\circledR}$ it is now possible to generate the so-called IDEAL 3D Model (Figure 13).

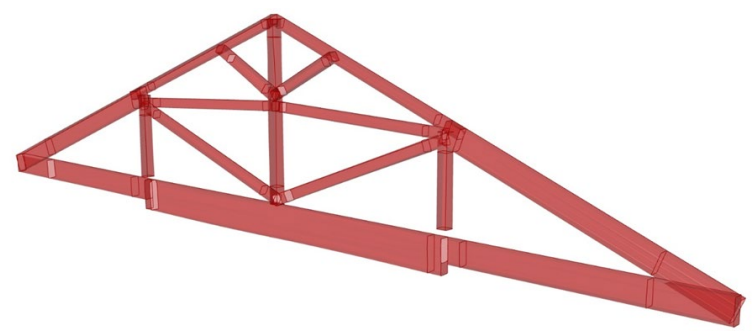

Figure 13: Ideal 3D model

\section{TRUSSES ANALYSIS THROUGH 3D MODELING AND POINT CLOUD COMPARISON}

The IDEAL 3D model of each truss can be compared to its clipped portion of the original point cloud in order to highlight their differences. As done before for analyzing the degree of compliance of the BASIC 3D model with the original point cloud, this step is carried out using the reverse engineering software Geomagic Control ${ }^{\circledR}$ that is able to examine and subsequently graphically report, in chromatic scale, the deviation between 3D model and point cloud. Minimum and maximum deviation threshold to be detected is set manually, in the chromatic scale, in order to have a better indication about the order of magnitude of dimensional differences between trusses. Geomagic Control ${ }^{\circledR}$ performs a comparative analysis to evaluate the deviations between the external faces of the solid geometric model and the point cloud. Graphical representation through the 
most appropriate chromatic scale makes clear the entities of the displacements. It is possible to select any position on every member of the truss in order to highlight locally the numerical value of deviations by means of a $2 \mathrm{D}$ graphical representation. This interpretation provides both qualitative and quantitative information and eases an accurate and widespread understanding of all movements and deformations that each truss underwent over time in the entire roofing. Moreover, lowerings and rotations of the king and queen posts can be analyzed and highlighted locally, as well as torsional phenomena or rotations of the tiebeams, lowering of bearings and bending of rafters, and eventually deformation phenomena withstanded by the whole roofing.

Comparisons can be made in a 3D mode (Figure 14) and on 2D projection planes selected by the user depending on the kind of truss member and on the type of deviations that have to be magnified. The software itself allows, in addition to deviations' graphical representation, their precise quantification using control points. By using the annotation tool, it is possible to report the exact value of the distance measured between the corresponding points of the two compared objects and its decomposition along the $\mathrm{x}, \mathrm{y}$ and $\mathrm{z}$ axes (Figure 15).
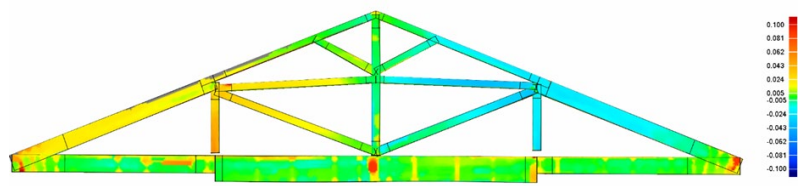

Figure 14: Three-dimensional representation of the displacement observed by overlapping the ideal 3D model and the point cloud of a single truss.

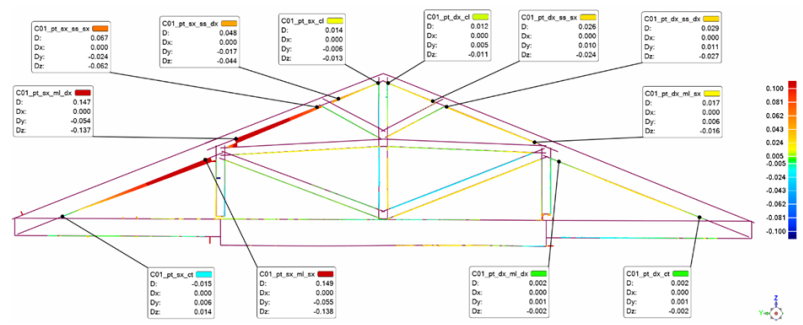

Figure 15: Bidimensional representation of the displacement observed by overlapping the ideal 3D model and the point cloud of a single truss. Annotation for rafters control points

Many types of movements or deviations can be highlighted by conveniently choosing these control points along some truss members constituting each truss under investigation. Precisely because of the way the ideal 3D model was modeled, it is possible to quantify, with enough precision, bending phenomena on principal rafters and on the tie-beam, rotations of posts in the truss plane, displacements outside the truss plane of rafters and posts.

Collected numerical data can be ordered in specific tables in order to obtain an overview of all values recorded through described analyses. Data collection thus becomes a very important step to ensure acquired information traceability and, once this information has been properly systematized, it becomes the tool through which to correctly interpret the behavior of these structural systems from a quantitative and not only qualitative point of view.
The methodology presented in this paper turned out to be an important step in the automatization of the whole research protocol. The analysis of the wooden roofing of St. Peter's Cathedral shows that its wooden trusses are in good condition, confirming the previous qualitative analysis. Moreover, the new numerical quantification makes it possible to compare these displacements and deformation with the safety limit values imposed by current regulation. In this way, the identification of truss members in structural problematic or risky condition becomes more precise and reliable.

This work, however, is not limited to the analysis of trusses in this particular case study but aims at the implementation of an equally efficient method applicable on every other similar type of wooden trusses into other roofings. The goal was to create a procedure that was as independent as possible from the operator who performs it.

Having an effective and efficient method available is an important goal, as it allows a more accurate study and understanding of these structures in a relatively timesaving and cost saving way. With these new available tools, it may be possible to design targeted and more effective restoration interventions.

The most significant result of what has been presented consists in the substitution of the manual work of rendering a 3D model with a process defined by generative algorithms. This substitution has brought a significant reduction in processing time and reduced the arbitrariness and inaccuracies due to the subjective considerations of the operator in the modeling phase. If the use of generative algorithm was already applied in the study of the Basilica of San Domenico, one of the most important enhancement of this work is the automatic generation of crosssections. This allows to completely avoid any manual and nonautomated intervention of the operator during the vectorizazion of curves from the point cloud. This highly elevates the repeatability and comparability of the proposed methodology in the future.

In addition to the rendering of the BASIC 3D model, it has been possible to define a series of algorithms able to generate automatically other 3D models by simply varying input parameters that seems to be an effective procedure for all structural systems with characteristics similar to wooden trusses. This means the possibility to render, in a short time and with good approximation, 3D models of large objects starting from a mere point cloud, even if incomplete and not totally closed.

Another very important result was achieved on the creation of the IDEAL 3D model that represents the truss at time zero, modeled without deformations and displacements. This model, crucial in the analysis of the behavior of the structure, has been rendered through a generation process, based on well-founded theoretical assumptions. The aim was to bring back the surveyed wooden truss in the conditions in which it should have been at construction time. In other words, remodeling perfectly linear and coplanar truss members, as they normally should be built.

Obviously it would be great (thus impossible) to have point cloud of the original roofing built in the XVII century. The IDEAL 3D model appears to be an effective way of reconstructing the original conditions of the roofing starting from the surveyed point cloud. In fact, it is generated by a procedure made of algorithms that works backwards, so as to bring the model back to an undeformed configuration.

Compared to previous works, where the ideal model was rendered as the average of all the surveyed trusses, the new method has eliminated most of the inaccuracies due to these approximations. Consequently, analyzes results can be considered most reliable and easily interpretable.

\section{CONCLUSIONS}




\section{FUTURE DEVELOPMENTS}

Good results obtained suggest applying this new method to other case studies already analyzed, in order to confirm both the best effectiveness in terms of quantitative definition of deviations and of evolution interpretation over time of wooden trusses, both the best efficiency in terms of working times and accuracy achieved. In the next future, the analysis through generative algorithm will be performed for the church of San Salvatore Maggiore and, hopefully, it will be possible to consider new case studies. The most interesting could be the Farnese Theater in which the roofing is built with wooden trusses of almost 32 meters of span. These trusses, although they are built in the middle of the XIX century, are known to be the widest still in place.

Apart from perfecting the research protocol by applying it to more case studies, other aspects can be considered for future developments:

1. Evaluation torsional phenomena present in the structural elements and possible implementation of another generative algorithm for their quantification;

2. Improvement of the results interpretation phase with the support of a detailed structural modeling directly using the rendered 3D models;

3. Deepening on the causes of deformations withstanded by the elements of the trusses over time through tests on materials;

The definition of an operative protocol consisting of defined and repeatable phases in a precise order can also allow, in the long term, the monitoring of the structure. Movements and deformations can be constantly tracked over time. This option allows to keep track, even after many years, of the changings the roofing underwent and to assess the state of conservation of wooden trusses. This deeper knowledge makes easier the design of timely targeted interventions to limit its degradation.

\section{REFERENCES}

3DSystems, 2014a. Geomagic Studio 2014, Version 2014.3.0. 3DSystems https://www.3dsystems.com/

3DSystems, 2014b. Geomagic Control 2014, Version 2014.3.0. 3DSystems https://www.3dsystems.com/

Bello Caballero, L., Mezzino, D., Federman, A., Santana Quintero, M., 2017. From field work to deliverables. Experiences on the tin house courtyard documentation. ISPRS - International Archives of the Photogrammetry, Remote Sensing and Spatial Information Sciences XLII-2/W5, 77-81. https://doi.org/10.5194/isprs-archives-XLII-2-W5-77-2017

Bertolini, C., Invernizzi, S., Marzi, T., Spanò, A.T., 2015. Numerical survey, analysis and assessment of past interventions on historical timber structures: the roof of Valentino Castle. Presented at the International Conference on Structural Health Assessment of Timber Structures, SHATIS'15, Dolnośląskie Wydawnictwo Edukacyjne, pp. 581-592.

Borsi, S., 1985. Giuliano da Sangallo: i disegni di architettura e dell'antico. Officina, Roma.

Émy, A.R., 1841. Traité de l'art de la charpenterie. Avanzo, New York.

Faro Technologies, 2018. Scene 2018, Version 2018.0.0.648 GA release Faro Technologies https://knowledge.faro.com/Software/FARO_SCENE

Guardigli, L., Lamborghini, S., Venturi, L., Mochi, G., 2015. Historic Timber Trusses In Europe. The Case Of St. Peter In
Bologna, in: Fifth International Construction History Congress. Construction History Society of America, Chicago, pp. 449-456.

James, M.R., Robson, S., Smith, M.W., 2017. 3-D uncertaintybased topographic change detection with structure-from-motion photogrammetry: precision maps for ground control and directly georeferenced surveys. Earth Surface Processes and Landforms 42, 1769-1788. https://doi.org/10.1002/esp.4125

Kalyan, T.S., Zadeh, P.A., Staub-French, S., Froese, T.M., 2016. Construction Quality Assessment Using 3D as-built Models Generated with Project Tango. Procedia Engineering 145, 1416 1423. https://doi.org/10.1016/j.proeng.2016.04.178

Lague, D., Brodu, N., Leroux, J., 2013. Accurate 3D comparison of complex topography with terrestrial laser scanner: Application to the Rangitikei canyon (N-Z). ISPRS Journal of Photogrammetry and Remote Sensing 82, 10-26. https://doi.org/10.1016/j.isprsjprs.2013.04.009

Munafò, P., 2002. Le capriate lignee antiche per i tetti a bassa pendenza: evoluzione, dissesti, tecniche di intervento. Alinea, Firenze.

Pizzagalli, F., Aluisetti, G., 1827. Dell'arte pratica del carpentiere esposta dagli architetti Felice Pizzagalli e Giulio Aluisetti. Gaspare Truffi, Milano.

Prati, D., Mochi, G., Guardigli, L., 2016. Contribution to the knowledge of wide span wooden roofing in the area of Bologna. Tema: Technology, Engineering, Materials and Architecture 2, 132-144. https://doi.org/10.17410/tema.v2i2.114

Prati, D., Rrapaj, I., Mochi, G., 2018. Contribution of parametric modeling in the interpretation of deformations and displacements of wooden trusses. SCIRES-IT - SCIentific RESearch and Information Technology 8, 105-120. https://doi.org/10.2423/i22394303v8n1p105

Robert Mcneel \& Associates, 2018a. Grasshopper algorithmic modeling for Rhino, Version 0.9.0076. Robert Mcneel \& Associates https://www.grasshopper3d.com/

Robert Mcneel \& Associates, 2018b. Rhinoceros 6, Version 6.3.18090.471. Robert Mcneel \& Associates https://www.rhino3d.com/it/

Scene 7.1 User's Manual, 2017. Faro Technologies https://knowledge.faro.com/Software/FARO_SCENE

Serlio, S., 1987. I sette libri dell'architettura. Arnaldo Forni, Sala Bolognese.

Valeriani, S., 2006. Kirchendächer in Rom: Beiträge zu Zimmermannskunst und Kirchenbau von der Spätantike bis zur Barockzeit $=$ Capriate ecclesiae, Berliner Beiträge zur Bauforschung und Denkmalpflege. Imhof, Petersberg.

Zamperini, E., 2015. Timber trusses in Italy: the progressive prevailing of open-joint over closed-joint trusses, in: Fifth International Construction History Congress. Construction History Society of America, Chicago, pp. 629-639.

Zamperini, E., 2013. The Study of Timber Structures Based on In Situ Investigation. Advanced Materials Research 778, 97-104. https://doi.org/10.4028/www.scientific.net/AMR.778.97 